$\mathrm{UK} / 11-06$

\title{
Quantum Quench across a Holographic Critical Point
}

\author{
Pallab Basu ${ }^{1}$ and Sumit R. Da: $2^{2}$ \\ Department of Physics and Astronomy, \\ University of Kentucky, Lexington, KY 40506, USA
}

\begin{abstract}
We study the problem of quantum quench across a critical point in a strongly coupled field theory using AdS/CFT techniques. The model involves a probe neutral scalar field with mass-squared $m^{2}$ in the range $-9 / 4<m^{2}<-3 / 2$ in a $A d S_{4}$ charged black brane background. For a given brane background there is a critical mass-squared, $m_{c}^{2}$ such that for $m^{2}<m_{c}^{2}$ the scalar field condenses. The theory is critical when $m^{2}=m_{c}^{2}$ and the source for the dual operator vanishes. At the critical point, the radial operator for the bulk linearized problem has a zero mode. We study the dynamics of the order parameter with a time dependent source $J(t)$, or a null-time dependent bulk mass $m(u)$ across the critical point. We show that in the critical region the dynamics for an initially slow variation is dominated by the zero mode : this leads to an effective description in terms of a Landau-Ginsburg type dynamics with a linear time derivative. Starting with an adiabatic initial condition in the ordered phase, we find that the order parameter drops to zero at a time $t_{\star}$ which is later than the time when $\left(m_{c}^{2}-m^{2}\right)$ or $J$ hits zero. In the critical region, $t_{\star}$, and the departure of the order parameter from its adiabatic value, scale with the rate of change, with exponents determined by static critical behavior. Numerical results for the order parameter are consistent with these expectations.
\end{abstract}

\footnotetext{
${ }^{1}$ e-mail:pallab.basu@uky.edu

${ }^{2}$ e-mail:das@pa.uky.edu
} 


\section{Contents}

1 Introduction and summary 2

2 Classical quench in a $0+1 \mathrm{dim}$ model 6

$2.1 J$ Quench ......................... 6

2.2 Mass quench . . . . . . . . . . . . . . . . . . 8

3 The equilibrium phase transition in the holographic model 10

4 Quench across the critical point the breakdown of adiabaticity. 13

4.1 Quenching the source . . . . . . . . . . . . . . 13

4.2 Scaling near the phase transition . . . . . . . . . . . . . . 17

4.3 Mass quench . . . . . . . . . . . . . . . . . . . . . . . . . 19

5 Numerics $\quad 20$

6 Outlook 21

A $\alpha=2$ quench

B $k$ dependence of coefficients : A toy model : 23

C $k$ dependence of the coefficients : the real problem 25

D Acknowledgements 


\section{$1 \quad$ Introduction and summary}

An important class of problems in quantum systems is that of quantum quench, where a parameter in the hamiltonian varies with time, typically attaining constant values at early and late times 3 . Starting with some initial state, the problem is to determine the nature of the final state. This problem has recently attracted a lot of attention in several areas of many-body physics, particularly because of progress in cold atom experiments [1], [2], [3]. Among other things, this problem is interesting for two different reasons. The first relates to the question of thermalization. Does the system evolve into some kind of steady state ? If so, is the state "thermal" in any sense? The second question deals with the situation where the quench takes place across a value of the parameter where there is an equilibriium critical point. In this case, Kibble-Zurek type scaling arguments indicate that there are several physical quantities which are universal and determined by the critical exponents of the critical point. Furthermore for two dimensional theories which are suddenly quenched to a critical point, powerful techniques of boundary conformal field theory have been used in [3] to show that ratios of relaxation times of one point functions, as well as the length/time scales associated with the behavior of two point functions of different operators are given in terms of ratios of their conformal dimensions at the critical point, and hence universal. Another related application of this phenomenon is in gravitational physics and cosmology. In this context, this is the phenomenon of particle production in time dependent backgrounds, which is relevant to many physical problems ranging from black hole evaporation to behavior of quantum fluctuations in an expanding universe.

There are very few theoretical tools available to study such systems when they are strongly coupled. In this note we will explore the use of AdS/CFT correspondence 44 - 7] to this problem. AdS/CFT techniques have been in fact used in the past to study quantum quench, though not at critical points. In AdS/CFT the boundary values of bulk fields become coupling constants, so that this problem becomes that of determining a time dependent background with specified initial and boundary conditions. As is usual, a quantum problem in the boundary theory becomes a classical problem in General Relativity.

One class of problems involve a vacuum initial state (in the bulk this is pure $A d S$ in the distant past) and a change of the boundary value of some field (e.g. the dilaton or the boundary metric) over some finite time interval, in a regime where supergravity is always valid. In the boundary field theory this corresponds to turning on a time dependent source

\footnotetext{
${ }^{3}$ Sometimes quench is used to denote a sudden change. We will use this word to denote changes with arbitrary rates, in particular slow rates.
} 
for the dual operator. Under suitable conditions, this leads to black hole formation in the bulk [8 10]. The correlators at future time would then be thermal with a temperature characterized by the Hawking temperature. The time scale after which this happens depends on the nature of the correlators, but turns out to be always smaller than what one would expect from a conformally invariant system evolving to a thermal state. Thus, in this case thermalization of the field theory is signalled by black hole formation. Another class of problems involve a similar setup, but a suitable variation of the coupling which prevents black hole formation in the supergravity regime. The coupling, however, becomes weak at some time and the bulk string frame curvature grows large, leading to a breakdown of the supergravity approximation - thus mimicking a space-like singularity [11]. For the case of a slow variation of the coupling it turns out that the gauge theory remains well defined and may be used to show that a smooth passage through this region of small coupling is possible without formation of a large black hole. Related scenarios appear in [12] and [13].

Many interesting phenomena in gauge-gravity duality, in particular phase transitions, can be explored consistently in a probe approximation. In this approximation, a certain set of bulk fields can be treated separately and independently of bulk gravity. One set of examples are probe branes in a background $A d S \times S$. This introduces hypermultiplets which in general live on a defect in the original field theory. When the number of such branes, $N_{f}$ is much smaller than the number of flux units which produce the background geometry, $N_{c}$, the dynamics of these branes do not backreact on the bulk metric which remains $A d S \times S$. The dynamics is then described by a DBI action in a fixed background - the fields in this action are then bulk probe fields. If the boundary value of such a probe field is time dependent in a fashion similar to above, black hole formation in the bulk is invisible in the probe approximation. However, rather remarkably, thermalization of the hypermultiplet sector is still visible. This manifests itself by the formation of an apparent horizon (which evolve into an event horizon in some situations) in the induced metric on the brane worldvolume 14, 15]. Fluctuations of the brane feel this induced metric and their correlators have thermal properties 4 .

None of the above works study quench dynamics near a phase transition. In this paper we take the first step towards doing that, in a simple model which displays an equilibrium critical point in a probe limit [19]. The model involves a neutral scalar field whose mass lies in the range $-9 / 4<m^{2}<-3 / 2$ in the background of a charged $A d S_{4}$ black brane. The overall coupling is chosen to be large so that the scalar can be treated as a probe. For a given brane background (i.e. for a given temperature and

\footnotetext{
${ }^{4}$ For related phenomena see 16
} 
charge density in the dual field theory), and for a vanishing non-normalizable mode of the field (corresponding to a vanishing source of the dual operator) there is always a critical mass $m_{c}^{2}$ below which the trivial solution is unstable. In this regime the stable solution is nontrivial, signifying a nonzero expectation for the dual operator in the dual theory. $m^{2}=m_{c}^{2}$ with a vanishing source is then a critical point, which happens to have standard mean field exponents. This setup is in fact quite similar to models of holographic superconductors and fermi surfaces [20]- 26]. However, here the scalar field is neutral so that the dynamics of the gauge field is frozen. This model has been argued to model phase transitions in antiferromagnets. As is well known, in this mass range there are two inequivalent quantizations. Our analysis is restricted to the conventional quantization of the bulk scalar, where the mode which vanishes slower at the boundary is treated as the source in the boundary theory. A similar analysis can be easily performed for the alternative quantization.

We will study the time evolution of the order parameter starting from adiabatic initial conditions in the ordered phase. The passage through the critical point is done in two ways - (i) by keeping the bulk mass at its critical value and changing the leading term of the near-boundary expansion of the bulk field and (ii) by changing a bulk mass parameter with a vanishing leading term. The field theory meaning of (i) is clear - this corresponds to turning on a source dual to the bulk field : the critical point appears when the source vanishes and the mass is at its critical value. The meaning of (ii) is less direct - changing a bulk mass means changing the anomalous dimension of the corresponding operator in the field theory. As discussed in [19], one way to do this is in fact coupling this scalar field to another field and changing the source for this other field.

Away from the critical point, adiabaticity holds for a sufficiently low rate of change. We will show that the adiabatic corrections are proportional to linear time derivatives, even though the equations of motion involve only second order time derivatives. This is a well-known phenomenon and happens because of dissipation implied by the presence of a horizon. In the bulk, we need to impose regularity conditions at the horizon, which is easily done using ingoing Eddington-Finkelstein coordinate [27,28] $u$ as time. $u$ coincides with the usual time coordinate $t$ on the boundary and $\partial /\left.\partial u\right|_{r}=\partial /\left.\partial t\right|_{r}$, though $\partial /\left.\partial_{r}\right|_{u} \neq \partial /\left.\partial r\right|_{t}$. The bulk equations now involve first order derivatives $\partial / \partial u$. As expected, adiabaticity fails as one approaches the critical point at a time which we choose to be $t=0$. The appearance of a first order time derivative is related to the fact that the dynamical critical

\footnotetext{
${ }^{5}$ Note that the analysis can be done using the usual time coordinate as well, as originally done in 29 , but one has to take special care of the behavior of the solution near the horizon. The result is of course the same.
} 
exponent of similar models turn out to be $z=2[30]$.

When adiabaticity fails, the system enters a scaling regime. A useful way to understand the dynamics is to decompose the bulk field in terms of eigenstates of the radial operator which appears in the linearized problem around the equilibrium solution. For any nonzero temperature of the background black brane, this operator has a zero mode which is regular at the horizon exactly at the critical point. We will show that in the critical region, the dynamics for small $v$ is dominated by that of this zero mode. The other modes remain adiabatic. The scaling properties can be then understood in terms of a Landau-Ginsburg type dynamics of the zero mode with a linear time derivative. The order parameter remains nonzero even when the instantaneous value of the coupling reaches the equilibrium critical value and first drops to zero at a time $t_{\star} \sim v^{-\alpha}$ where $v$ denotes the rate of change of the coupling in the critical region. The departure of the value of the order parameter from the instantaneous equilibrium value also scales as $v^{\beta}$. The exponents $\alpha$ and $\beta$ are essentially determined by the static critical exponents.

Beyond the critical region, however, the other modes become important. In fact, at very late times the bulk equation may be approximated by its linearized form, so that the solutions are nothing but the usual quasinormal modes around the background black hole. As usual, the imaginary part of the lowest quasinormal mode frequency then determine the decay of late time behavior.

We also present preliminary results of a numerical solution of the time dependent equations for the bulk field and extract the time dependence of the order parameter. The results are consistent with the above picture. Our numerical results are not yet accurate enough to verify the scaling behavior. However this should be possible with further work.

In Section 2 we discuss spatially homogeneous classical quench in a Landau-Ginsburg type model as a prelude. In Section 3 we review the main faetures of the equilibrium phase transition. In Section 4 we discuss the failure of adiabaticity in quantum quench across this transition. We then show that in the critical region the dynamics for an initially slow quench is dominated by that of the zero mode, and discuss its relationship of the dynamics to the Landau-Ginsburg model of Section 2. In Section 5 we present the results of a numerical solution of the bulk equation of motion and verify the expectations in the previous section. Section 6 contains some concluding remarks. The three appendices provide details of derivation of the some of the results contained in the main text. 


\section{Classical quench in a $0+1 \mathrm{dim}$ model}

As a prelude to the main discussion, in this section we will study classical spatially homogeneous quench in a Landau-Ginsburg model, which is described by the equation,

$$
\frac{d^{\alpha} \phi}{d t^{\alpha}}+m^{2}(t) \phi+\phi^{2 \beta-1}+J(t)=0
$$

As discussed above, holography converts the problem of a quantum quench to a problem of classical quench in one more dimension - which is the motivation behind studying (2.1). In a later section we will show that this simple model describes some of the essential features of the dynamics in the critical region of the holographic phase transition which is the main subject of this paper. Note that we do not have any noise term (which is what is needed for many other applications).

When $m^{2}$ and $J$ are time independent, this model has a critical point at $m^{2}=J=0$. Here we have kept generic $\alpha$ and $\beta$ for convenience of later discussion. In most conservative models, i.e. where energy is conserved, $\alpha=2$. However as we will see $\alpha=1$ is more appropriate for the holographic transition which we will discuss. In the following we will therefore use this value. We will also concentrate on the $\beta=2$ case, i.e. a quartic interaction.

\section{$2.1 \quad J$ Quench}

Let us first discuss the case where $J(t)$ is time varying, but a $m^{2}$ which is independent of time and positive.

For a time independent $J$ we have,

$$
m^{2} \phi+\phi^{3}+J=0
$$

Denote the solution of this static equation by $\phi_{0}(J, m)$. We then introduce a $J(t)$ which is slowly varying with time. The idea is to start with adiabatic initial conditions at some early enough time and study the time evolution of the order parameter. To study the adiabatic expansion we write,

$$
\phi=\phi_{0}(J(t), m)+\epsilon \phi_{1}(t)+\cdots
$$

Here $\epsilon$ is an adiabaticity parameter introduced by performing a scaling $t \rightarrow t / \epsilon$ so that any $\partial_{t}$ comes with a factor of $\epsilon$. The ellipsis denote higher order terms in $\epsilon$. To lowest order in $\epsilon$, the equation governing $\phi_{1}$ is,

$$
\phi_{1}\left(m^{2}+3 \phi_{0}^{2}(t)\right)=\dot{\phi}_{0}(t)=\dot{J}(t) \frac{\partial \phi_{0}}{\partial J}
$$


The adiabatic expansion is good for a finite $m, \phi_{0}$ for a sufficiently slowly varying $J(t)$. The adiabatic expansion fails when

$$
\begin{aligned}
& \phi_{1} \sim \phi_{0} \\
\Rightarrow & \frac{1}{m^{2}+3 \phi_{0}^{2}} \dot{\phi}_{0} \sim \phi_{0}
\end{aligned}
$$

For a sufficiently slowly varying $J(t)$, this is possible if both $m^{2}$ and $\phi_{0}$ are small, i.e. when we are close to the critical point $m^{2}=J=0$. Approaching the critical point along the direction $m^{2}=0$ we have $\phi_{0}(t) \approx(-J(t))^{\frac{1}{3}}$, and 2.6 leads to the condition for breakdown of adiabaticity ,

$$
\dot{J} \sim J^{\frac{5}{3}}
$$

If the quench is linear near the critical point, that is $J(t) \sim J_{0} v t$ for small $J$, we get

$$
v t^{\frac{5}{2}} \sim 1
$$

as the condition for breakdown of adiabaticity.

When adiabaticity breaks down, the system enters a scaling region, In the scaling region we use $J(t)=J_{0} v t+O\left(t^{2}\right)$. We rescale the field as: $\phi \rightarrow \alpha \tilde{\phi}$ and $t \rightarrow \beta \tilde{t}$. Choosing $\alpha^{2} \beta=1$ and $v \frac{\beta^{2}}{\alpha}=1$, we get a $v$ independent equation for $\tilde{\phi}(\tilde{t})$. The quadratic or higher order temporal contribution in $J(t)$ only gives a sub-leading contribution in $J_{0} v$ in the scaling limit. Hence we have a scaling solution,

$$
\phi(t)=v^{\frac{1}{5}} \tilde{\phi}\left(v^{\frac{2}{5}} t\right)
$$

This scaling solution leads to an estimate of the magnitude of fluctuations $(\delta \phi(0))$, i.e. the departure of $\phi$ from the equilibrium value at $t=0$. Outside the critical region $\delta \phi(0) \sim \frac{v}{m^{2}}$, whereas in the critical region $\delta \phi(0) \sim v^{\frac{1}{5}}$. This also defines the "zero crossing time" $\left(t_{\star}\right)$ defined by $\phi\left(t_{\star}\right)=0$. We have $t_{\star} \sim v^{-\frac{2}{5}}$ at the critical point. Which diverges in the $v \rightarrow 0$ limit. Out of criticality $t_{\star}$ actually approaches a constant in the $v \rightarrow 0$ limit.

It is instructive to study the dynamics of the order parameter for a profile of $J(t)$ which becomes a constant at early and late times and behaves linearly with time in the critical region, for example

$$
J(t)=J_{0} \tanh (v t)
$$

with $m^{2}=0$ for all times. The numerical solution of the equation of motion with an adiabatic initial condition at early times is shown in Figure (2.1) for a typical value of $v$.

Numerically we see that at sufficiently early times the order parameter tracks the adiabatic solution and overshoots the adaiabatic solution as one approaches the critical 


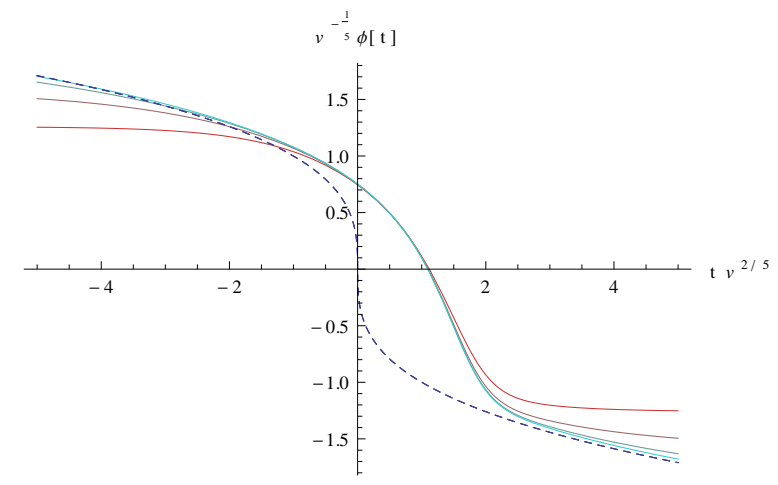

Figure 1: The order parameter as a function of time for a $J(t)=\tanh (v t)$ at $m^{2}=0$ with $v=10^{-0.5}, 10^{-1}, 10^{-1.5}, 10^{-2}$ (from the bottom on the left). The adiabatic solution (dashed) is also shown as a comparison.

region at $t=0$, falling down to zero at some positive time $t_{\star}$. At later times, the solution again approaches the adiabatic solution. The numerical solutions for different values of $v$ are consistent with the behavior $\delta \phi(0) \sim v^{\frac{1}{5}}$ and $t_{\star} \sim v^{-\frac{2}{5}}$ as predicted by the scaling analysis.

For profiles of $J(t)$ which behave as some nontrivial power of $t$ in the critical region, i.e. $J(t) \sim(v t)^{n}$ a similar analysis leads to

$$
\phi(t, v)=v^{\frac{n}{2 n+3}} \tilde{\phi}\left(t v^{\frac{2 n}{2 n+3}}, 1\right)
$$

leading to the scaling properties

$$
\delta \phi(0) \sim v^{\frac{n}{2 n+3}} \quad t_{\star} \sim v^{-\frac{2 n}{2 n+3}} .
$$

If there are more than one interaction terms, e.g. $\phi^{2 \beta-1}$ present in the equation of motion, then the lowest order interaction $\left(\beta_{0}\right)$ dominates the critical dynamics. In this case we have,

$$
\phi(t, v)=v^{\frac{n}{\left(2 \beta_{0}-2\right)(n+1)+1}} \tilde{\phi}\left(t v^{\frac{\left(2 \beta_{0}-2\right) n}{(n+1)\left(2 \beta_{0}-2\right)+1}}\right) .
$$

\subsection{Mass quench}

In this section we will study classical quench across the critical point along the line $J=0$ by changing $m^{2}$ from negative to positive values. For static $m^{2}<0$, the model has a non-trivial time-independent solution $\phi_{0}=\sqrt{-m^{2}}$. The static model has a second order phase transition near $m^{2}=0$. Now we introduce a time dependent mass function which 
asymptotes to some constant value of $m^{2}<0$ at early times and then rise to a constant value $m^{2}>0$ at late times, e.g.

$$
m^{2}(t)=-m_{1}^{2} \tanh (v t)
$$

Once again we start with adiabatic initial conditions at some early enough time and study the time evolution of the order parameter. To study the adiabatic expansion we write,

$$
\phi=\phi_{0}(t)+\epsilon \phi_{1}(t)+\cdots
$$

where,

$$
\phi_{0}(t)=\sqrt{-m^{2}(t)}
$$

To lowest order in the adiabatic expansion

$$
\phi_{1}=\frac{\dot{\phi}_{0}}{2 m^{2}}
$$

Using the fact that $\phi_{0} \sim \sqrt{\left.-m^{2}\right)}$, we get that the adiabatic expansion breaks down as $\phi_{1} \sim \phi_{0}$, i.e. when

$$
\frac{\sqrt{\left(-m^{2}\right)}}{\left(-m^{3}\right)} \sim 1 .
$$

As expected this breaks down as we approach the equilibrium critical point. If $(-m)^{2} \approx v t$ near the critical point breakdown of adiabaticity happens when,

$$
t \propto v^{-\frac{1}{2}}
$$

When adiabaticity breaks down the system enters a scaling regime. In this regime, we can approximate $m^{2}(t)=-m_{1}^{2} v t$. Here the scaling relations are

$$
\phi(t, v) \approx v^{\frac{1}{4}} \tilde{\phi}\left(t v^{\frac{1}{2}}, 1\right)
$$

However, if the solution does not cross a phase transition point then fluctuation around a static solution is suppressed exponentially in $\frac{1}{v}$. It is also to be noted that if $m(t)$ is sufficiently slowly varying, the exact functional form of $m(t)$ is not needed. The only information we use to derive 2.20 is that $m(t)^{2}$ is a linear function of time near the phase transition and $m(t) \rightarrow m_{2}$ as $t \rightarrow \infty$. 


\section{The equilibrium phase transition in the holographic model}

The model of [19 has a neutral scalar field $\phi(t, r, \vec{x})$ in the background of a charged $A d S_{4}$ black brane. The lagrangian is given by

$$
\mathcal{L}=\frac{1}{2 \kappa^{2} \lambda} \sqrt{-g}\left[-\frac{1}{2}(\partial \phi)^{2}-\frac{1}{4}\left(\phi^{2}+m^{2}\right)^{2}-\frac{m^{4}}{4}\right]
$$

The background metric is given by (in $R_{A d S}=1$ units)

$$
d s^{2}=\left[-r^{2} f(r) d t^{2}+r^{2} d \vec{x}^{2}\right]+\frac{d r^{2}}{r^{2} f(r)}
$$

where

$$
f(r)=\left[1+\frac{3 \eta r_{0}^{4}}{r^{4}}-\frac{1+3 \eta r_{0}^{3}}{r^{3}}\right] \quad 0 \leq \eta \leq 1
$$

The associated Hawking temperature is then given by

$$
T=\frac{3}{4 \pi r_{0}}(1-\eta)
$$

In the following we will replace $r \rightarrow r r_{0}$.

One should add the Einstein-Hilbert action and a cosmological constant term to 3.21. In the limit of large $\lambda$, however, the field $\phi$ can be regarded as a probe field since its back reaction to the metric via Einstein's equations is small. This is the approximation we adopt.

In [19] it was shown that when the mass lies in the range

$$
-\frac{9}{4}<m^{2}<-\frac{3}{2}
$$

there is a critical phase transition at some value of $T=T_{c}(m)$ when the source to the dual operator vanishes. Conversely, for a given $T$ there is a value of $m^{2}=m_{c}^{2}$ where the theory is critical.

The upper limit in 3.25 is the BF bound for the near-horizon $A d S_{2}$ geometry which appears in the extremal $(\eta=0)$ metric. (Note that the AdS scale for this infrared $A d S_{2}$ is given by $1 / \sqrt{6}$ in our units). The lower bound is the BF bound for the asymptotic $A d S_{4}$. Field configurations which are translationally invariant in the $\vec{x}$ directions satisfy the equations of motion

$$
\frac{1}{r^{2}}\left[-\frac{r^{2}}{f(r)} \partial_{t}^{2}+\partial_{r}\left(r^{2} f(r) \partial_{r}\right)\right] \phi-m^{2} \phi-\phi^{3}=0
$$


Near the $A d S_{4}$ boundary the asymptotic behavior of the solution to the linearized equation is of the form 6

$$
\phi(r)=J(t) r^{-\Delta_{-}}\left[1+O\left(1 / r^{2}\right)\right]+<\mathcal{O}>(t) r^{-\Delta_{+}}\left[1+O\left(1 / r^{2}\right)\right]
$$

where $\Delta$ is given by

$$
\Delta_{ \pm}=\frac{3}{2} \pm \sqrt{m^{2}+\frac{9}{4}}
$$

In the range of masses of interest, both the solutions are normalizable, so that there is a choice of quantization. The standard quantization considers the coefficient $J(t)$ is the source in the dual field theory and $\langle\mathcal{O}\rangle(t)$ then gives the expectation value of the dual operator. In the alternative quantization the expectation and source change the role.

Consider first the linearized problem, ignoring the cubic term. By a standard change of coordinates to tortoise coordinates $\rho$ and a field refinition to $\chi$,

$$
d \rho=-\frac{d r}{r^{2} f(r)} \quad \phi(r, t)=\frac{\chi(\rho, t)}{r}
$$

The horizon is then at $\rho=\infty$ and the boundary is at $\rho=0$. the equation becomes $([24])$

$$
-\partial_{t}^{2} \chi=-\partial_{\rho}^{2} \chi+V_{0}(\rho) \chi \equiv \mathcal{P}_{\rho} \chi
$$

with

$$
V_{0}(\rho)=f(r)\left[\left(m^{2}+2\right)-\frac{6 \eta}{r^{4}}+\frac{1+3 \eta}{r^{3}}\right]
$$

where in $V_{0}(\rho)$ we need to express $r$ in terms of $\rho$ using (3.29).

For solutions of the type $\chi \sim e^{-i \omega t}$, equation 3.31 is a Schrodinger problem in a potential $V(\rho)$. The potential goes to zero at the horizon $\rho=-\infty$ and behaves as $\frac{\left(m^{2}+2\right)}{\rho^{2}}$ near the boundary $\rho=0$. Note that for a brane background at any finite temperature, $f(r) \sim(r-1)$ near the horizon, while $\rho \sim-\log (r-1)$ so that $V_{0} \sim e^{-\rho}$ as we approach the horizon. In contrast, for the extremal background $f(r) \sim(r-1)^{2}$ while $\rho \sim 1 /(r-1)$ so that $V_{0} \sim 1 / \rho^{2}$. This makes the analysis for the extremal background rather subtle. In this paper we will work with the non-extremal case.

Due to the vanishing of the potential near the horizon the time-independent problem has a continuum of modes starting at $\omega=0$. The behavior of the solution near the horizon $\rho=-\infty$ is of the form $e^{-i \omega(t \pm \rho)}$, the two signs representing ingoing and outgoing waves. We are eventually interested in solving the problem with ingoing boundary conditions at the horizon.

\footnotetext{
${ }^{6}$ When we turn on $J(t)$, it is a valid concern whether we will be able to neglect the non-linear term near the boundary. This can be done as long as $\Delta>0$ or $m^{2}<0$.
} 


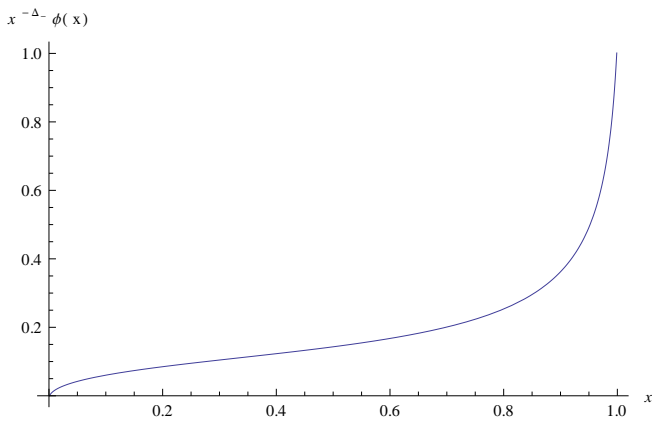

Figure 2: Profile of (rescaled) zero mode $\left(x^{-\Delta_{-}} \phi(x)\right.$, where $\left.\left.x \sim \frac{1}{r}\right)\right)$ with $m^{2} \approx-2.235$ and $\eta=0.98$.

Generically for any value of $m^{2}$ there are quasi-normal modes with complex frequencies. These are normalizable modes with ingoing boundary condition. Unlike the continuum discussed above these modes form a set of disconnected poles in the complex frequency plane. However, normalizable bound states appear when $m^{2}$ is sufficiently negative : this is what has been shown in [19].

There is a critical value of $m^{2}=m_{c}^{2}$ when a zero energy bound state appears, which vanishes in an appropriate fashion at the boundary and is in addition purely ingoing at the horizon. In the complex frequency plane some quasinormal mode(s) hit the origin at $m^{2}=m_{c}^{2}$. This critical mass is $m_{c}^{2}=-\frac{3}{2}$ when $\eta=1$ and decreases with decreasing $\eta$ or increasing temperature. The existence of such a bound state for $m^{2}=-2$ has been shown by variational methods in [21]. A similar demosntration should be possible for other values of $m^{2}$. We have numerically verfied the existence of this zero mode. A typical plot the zero mode is shown in in Fig 2. Note that the leading large-r behavior has been factored out to ensure that the source term indeed vanishes at the boundary.

The presence of a bound state in the Schrodinger problem means that the solution $\chi=0$ of the full nonlinear problem is unstable, and one has to look for other nontrivial solutions. In [19] it was shown that such a stable nontrivial static solution $\phi_{0}(r)$ exists in the range of masses given by (3.25), both for the standard and alternative quantizations. This means that the expectation value of the dual operator is nonzero, i.e. the field condenses, and $m^{2}=m_{c}^{2}(T)$ is a critical point . One can approach this critical point either by fixing the mass and tuning the temperature, or by fixing the temperature and tuning the mass. The latter may appear to be somewhat strange from the point of view of the boundary field theory. However, it is only the IR mass which is relevant - and one may imagine obtaining this by coupling $\phi$ to another field $\Psi$ by $\Psi \phi^{2}$. The expectation 
value of $\Psi$ which may results from changing a coupling in the boundary field theory then renders a mass to $\phi[19$.

The critical behavior has standard mean field exponents at any finite $T$. If the operator dual to the field $\phi$ is $\mathcal{O}$ and the source is $J$ then an analysis identical to that presented in 19 leads to (for $m^{2}-m_{c}^{2} \rightarrow 0^{+}$)

$$
<\mathcal{O}>\left._{J=0} \sim\left(m^{2}-m_{c}^{2}\right)^{1 / 2} \quad \frac{d<\mathcal{O}>_{J=0} \sim\left(m^{2}-m_{c}^{2}\right)^{-1}}{d J}\right|_{J} \quad<\mathcal{O}>_{m=m_{c}} \sim J^{1 / 3}
$$

Exactly at zero temperature the phase transition is of BKT type and the order parameter depends exponentially

$$
<\mathcal{O}>_{J=0} \sim \exp \left[-\frac{\pi \sqrt{6}}{2 \sqrt{m_{c}^{2}-m^{2}}}\right]
$$

The zero mode of the linearized operator will play a key role in the following. This zero mode is known to exist for any non-zero temperature. At zero temperature the situation is less clear. For a similar zero temperature system involving the D3-D5 system [32] such a zero mode does not exist 33 .

\section{Quench across the critical point the breakdown of adiabaticity.}

Our aim is to study quench dynamics across this equlibrium phase transition. In a quench situation, some parameter of the boundary theory is made time dependent. The first issue we will study is the breakdown of adiabaticity as we approach the critical point. First, we argue that away from the critical point, the order parameter tracks the changing coupling adiabatically. We will then determine the regime near the critical point where adiabataicity breaks and show that in this region the order parameter and the time scale are proportional to some power of $v$ pretty much like the model studied in section 2 .

\subsection{Quenching the source}

As discussed above, a simple way to quench across the critical point is to remain at the critical value of the mass and consider a time dependent source for the dual operator in the boundary field theory. This corresponds to a nontrivial boundary value for the bulk field. Our discussion is in the conventional quantization of the theory, i.e $J(t)$ in (3.27) is treated as a source. The case of alternative quantization should follow along identical lines. 
It is well known that to study low frequency modes in the background of a black brane it is convenient to use ingoing Edddington-Finkelstein coordinates,

$$
u=\rho-t, \quad \rho
$$

where $\rho$ is defined in 3.29 . In terms of these coordinates the equation of motion 3.26 becomes

$$
-2 \partial_{u} \partial_{\rho} \chi=-\partial_{\rho}^{2} \chi+V(\rho, \chi)
$$

where

$$
V(\rho, \chi)=V_{0}(\rho) \chi+\frac{f(r)}{r^{2}} \chi^{3} .
$$

This equation has to be solved with the boundary condition that the field is regular at the horizon, which at the linearized level is equivalent to requiring that the waves are purely ingoing at the horizon 27, 28. At the linear level the analysis can be of course performed in $(t, \rho)$ coordinates as well. However, in this case one needs special care to extract a leading singular piece before performing the low energy expansion [29]. At the full nonlinear level this procedure is possibly rather involved, but imposing regularity in EF coordinates remain simple.

We need to solve 4.35 with the condition

$$
\chi(u, \rho) \rightarrow \rho^{-1+\Delta_{-}} J(u) \quad \text { as } \rho \rightarrow 0
$$

where $\Delta_{ \pm}$are defined in 3.28 . We have considered the source to be a function of $u$ rather than a function of $t$. On the boundary $\rho=0 \rho$ and $t$ are the same (and quite generally $\partial /\left.\partial u\right|_{\rho}=\partial /\left.\partial t\right|_{\rho}$ ), so that this does correspond to a time dependent source in the boundary theory. However, as we will soon see, it is convenient to use 4.37). This form makes bulk causality explicit.

To perform the adiabatic expansion, let us decompose the field $\chi(\rho, u)$ as

$$
\chi(\rho, u)=\chi_{l}(\rho, u)+\chi_{s}(\rho, u)
$$

Where $\chi_{l}(\rho, u)=J(u) \rho^{-1+\Delta_{-}}$and $\chi_{s}(\rho, u) \sim \rho^{-1+\Delta_{+}}$as $\rho \rightarrow 0$. We get,

$$
\begin{array}{r}
{\left[-\partial_{\rho}^{2}+V_{0}(\rho)\right] \chi_{s}+\frac{f(r)}{r^{2}}\left(\chi_{s}^{3}+3 \chi_{l} \chi_{s}^{2}+3 \chi_{l}^{2} \chi_{s}\right)=-\left[-\partial_{\rho}^{2}+V_{0}(\rho)\right] \chi_{l}-\frac{f(r)}{r^{2}} \chi_{l}^{3}} \\
-2 \partial_{u} \partial_{\rho} \chi_{l}-2 \partial_{u} \partial_{\rho} \chi_{s}
\end{array}
$$

If $\chi_{l}(\rho, u)=\chi_{l}(\rho)$ is time independent we have a static solution $\chi_{s}(\rho, u)=\chi_{0}(\rho)$. This has been discussed in the previous section. When we turn on a time dependent $J(u)$ 
which is slowly varying, one expects that the time dependent solution of 3.26 may be constructed in an adiabatic expansion. To understand that we write,

$$
\chi_{s}(\rho, u)=\chi_{0}(\rho, J(u))+\epsilon \chi_{1}(\rho, u)+\cdot
$$

Here $\epsilon$ is an adiabaticity parameter which keeps track of the adiabatic expansion. If we scale $u \rightarrow u / \epsilon$, each $u$ derivative is of order $O(\epsilon)$. The ellipsis in (4.62) are higher order terms in $\epsilon$. The idea then is to insert 4.62 into the equations of motion and obtain equations for $\chi_{1}, \chi_{2}, \cdots$ order by order in $\epsilon$. To the lowest order one gets

$$
\mathcal{D}_{\rho}^{(1)} \chi_{1}=\left\{\left[-\partial_{\rho}^{2}+V_{0}(\rho)\right]+\frac{f(r)}{r^{2}}\left(3 \chi_{0}^{2}+6 \chi_{l} \chi_{0}+3 \chi_{l}^{2}\right)\right\} \chi_{1}=+2 \partial_{u} \partial_{\rho} \chi_{l}+2 \partial_{u} \partial_{\rho} \chi_{0}
$$

In deriving 4.41 we have ignored any time dependence of $\chi_{1}$, as is appropriate in an adiabatic expansion. Generically, at each order in perturbation we may cast the adiabatic problem as,

$$
\mathcal{D}_{\rho}^{(n)} \chi_{n}=\mathcal{J}_{n}(u, \rho)
$$

where $\mathcal{J}_{n}(u)$ is a source term contains time derivatives and determined by the lower order equations in an adiabatic expansion.

As discussed before, near the black hole horizon the differential operator becomes a plane wave operator and the potential part vanishes. Since the potential $V_{0}(\rho)$ goes to zero at the horizon $\rho=\infty$ and the terms which involve $\chi_{0}, \chi_{l}$ in 4.41 also vanish at the horizon (because of the overall factor of $f(r)$ ), the operator $\mathcal{D}_{\rho}$ has a continuous spectrum which begins at zero. It is, therefore, a non-trivial fact that there is adiabaticity away from the critical point. We will now show why this is so and how adiabaticity breaks down near the critical point.

These equations are most conveniently solved by Green's function method,

$$
\chi_{n}=\int d \rho^{\prime} G^{(n)}\left(\rho, \rho^{\prime}\right) \mathcal{J}_{n}\left(u, \rho^{\prime}\right) .
$$

where $G^{(n)}\left(\rho, \rho^{\prime}\right)$ is the Green's function of the operator $\mathcal{D}_{\rho}^{(n)}$ with the boundary conditions $G(0, \rho)=0$ and $G(\infty, \rho)$ is regular.

Let us concentrate on the lowest order terms in the adiabatic expansion, $n=1$. All higher terms may be found out by similar procedure. The Green's function is given by,

$$
\begin{array}{rlr}
G^{(1)}\left(\rho, \rho^{\prime}\right) & =\frac{1}{W\left(\chi_{1}, \chi_{2}\right)} \chi_{1}\left(\rho^{\prime}\right) \chi_{2}(\rho), \quad & \rho<\rho^{\prime} \\
& =\frac{1}{W\left(\chi_{1}, \chi_{2}\right)} \chi_{2}\left(\rho^{\prime}\right) \chi_{1}(\rho), \quad \rho>\rho^{\prime},
\end{array}
$$


where $\chi_{1}$ and $\chi_{2}$ are solutions of homogeneous part of eqn 4.42 satisfying appropriate boundary condition at the horizon $\rho=\infty$ and the boundary $\rho=0$ respectively, and $W\left(\chi_{1}, \chi_{2}\right)$ is the Wronskian which is independent of $\rho$ in this case. We have normalized $\chi_{1}(\rho)$ and $\chi_{2}(\rho)$ in such a fashion that $\chi_{1}=1$ at the horizon and $\chi_{2} \rightarrow \rho^{-1+\Delta_{-}}$near the boundary.

Given that we are looking for a solution which is regular at the horizon $(\rho \rightarrow \infty), \chi_{1}$ is a constant at the horizon and so is the Green's function. Nevertheless, the integral in eqn (4.43) is is finite. This is because regularity of the functions $\chi_{l}$ and $\chi_{0}$ mean that $\partial_{r} \chi_{l}, \partial_{r} \chi_{0}$ are finite at the horizon. Since $(r-1) \sim e^{-\rho}$, this implies that $\mathcal{J}(u, \rho) \sim$ $\partial_{\rho}\left(\chi_{l}+\chi_{0}\right) \sim \exp (-\rho)$, damping out any divergence in 4.43 from the large $\rho$ region.

In general, near the horizon $\chi_{2}$ can be expressed as a linear combination of a regular and irregular solution, i.e $\chi_{2}(\rho \rightarrow \infty)=a \rho+b$. It can be easily checked that $W\left(\chi_{1}, \chi_{2}\right)=$ a. Hence,

$$
\chi_{1}(\rho, u)=-\frac{1}{a} \int d \rho^{\prime}\left[\theta\left(\rho-\rho^{\prime}\right) \chi_{1}\left(\rho^{\prime}\right) \chi_{2}(\rho)+\left(\rho \rightarrow \rho^{\prime}\right)\right]\left(2 \partial_{u} \partial_{\rho} \chi_{0}+2 \partial_{u} \partial_{\rho} \chi_{l}\right)
$$

This is finite as long as $a$ is finite, and small for sufficiently small $\partial_{u} \chi_{0}$ and $\partial_{u} \chi_{l}$.

At the phase transition point, however, the operator $\mathcal{D}_{\rho}^{(1)}$ has a zero mode which is regular at the horizon. This is because at $J=0$ the factors involving $\chi_{0}$ and $\chi_{l}$ in the left hand side of (4.41) vanish, and the operator is identical to the operator acting on the linearized small fluctuations at $m^{2}=m_{c}^{2}$ around the trivial solution $\chi_{0}=0$, i.e. the operator $\mathcal{P}_{\rho}$ which appears on the right hand side of $(3.30)$. We know that this operator has a zero mode which is regular at the horizon and vanishes as $\rho^{-1+\Delta_{-}}$at the boundary $\rho=0$. This means that at this point $a=0$. Therefore, the first adiabatic correction diverges.

To derive the precise condition for adiabaticity, it is sufficient to look at the operator $\mathcal{D}_{\rho}^{(1)}$ for small $J(u)$. For small $J(u)$, the leading departure from the critical operator comes from the term which is proportional to $\chi_{0}^{2} \sim J^{2 / 3}$. Thus we can use perturbation theory in $J$ to estimate $a \propto J^{2 / 3}$. As argued before, $\chi_{0} \sim(-J)^{\frac{1}{3}}$, while $\chi_{l} \sim J$. Hence the leading divergence in $\chi_{1}$ can be estimated as

$$
\chi_{1}(\rho, u) \sim \frac{J^{-2 / 3} \dot{J}}{J^{2 / 3}}=J^{-4 / 3} \dot{J} .
$$

The breakdown of adiabaticity happens when,

$$
\begin{gathered}
\chi_{1}(\rho, u) \sim \chi_{0} \\
\Rightarrow \dot{J} \sim J^{5 / 3},
\end{gathered}
$$


exactly as in the $0+1$ dimensional model (equation (2.7). Assuming a linear quench, i.e $J(u) \approx v u$, we get

$$
v u^{\frac{5}{2}} \sim 1
$$

as the condition for breakdown of adiabaticity.

\subsection{Scaling near the phase transition}

In this section we argue that the critical region dynamics is dominated by that of the zero mode for (initially) small quench rate. We will demonstrate this for the case where $J(u)=v u$ in the critical region. Extension to non-linear quenches is straightforward.

To understand this let us rescale $\chi \rightarrow v^{\frac{1}{5}} \tilde{\chi}_{s}, u \rightarrow v^{-\frac{2}{5}} \tilde{u}$.. Assuming a linear quench, i.e. $\chi_{l} \sim v u \tilde{\chi}_{l}(\rho)$, we have at the leading order in $v$,

$$
\left[-\partial_{\rho}^{2}+V_{0}(\rho)\right] \tilde{\chi}_{s}+v^{\frac{2}{5}}\left[\frac{f(r)}{r^{2}}\left(\tilde{\chi}_{s}\right)^{3}+\tilde{u}\left[-\partial_{\rho}^{2}+V_{0}(\rho)\right] \tilde{\chi}_{l}+2 \partial_{\tilde{u}} \partial_{\rho} \tilde{\chi}_{s}\right]+\cdots=0
$$

The ellipsis denote terms which contains higher powers of $v$.

Now let us expand the sub-leading part of the scalar field in eigenfunctions of the operator $\mathcal{P}_{\rho}$ (defined in equation $(3.30)$ at the critical point,

$$
\tilde{\chi}_{s}(\rho, u)=\int \tilde{a}_{k}(u) \chi_{k}(\rho) d k
$$

where $\chi_{k}$ satisfy

$$
\mathcal{P}_{\rho}^{c} \chi_{k}=\left[-\partial_{\rho}^{2}+V_{0}^{c}(\rho)\right] \chi_{k}=k^{2} \chi_{k}
$$

where $V_{0}^{c}$ denotes the potential in (3.31) at $m^{2}=m_{c}^{2}$. the eigenfunctions $\chi_{k}(\rho)$ are delta function normalized. The eigenfunctions obey the condition

$$
\operatorname{Lim}_{\rho \rightarrow 0}\left[\rho^{-\Delta_{-}} \chi_{k}(\rho)\right]=0
$$

In terms of the eigen-coefficients $a_{k}(u)$ the equation 4.51) becomes,

$$
k^{2} \tilde{a}_{k}+v^{\frac{2}{5}}\left(\tilde{u} \mathcal{J}_{k}-\int b_{k k^{\prime}} \partial_{\tilde{u}} \tilde{a}_{k^{\prime}} d k^{\prime}-\int \tilde{a}_{k^{\prime}} \tilde{a}_{k^{\prime \prime}} \tilde{a}_{k^{\prime \prime \prime}} C_{k, k^{\prime}, k^{\prime \prime}, k^{\prime \prime \prime}} d k^{\prime} d k^{\prime \prime} d k^{\prime \prime \prime}\right)=0
$$

where,

$$
\begin{gathered}
\mathcal{J}_{k}=\int \chi_{k}(\rho)\left[-\partial_{\rho}^{2}+V_{0}(\rho)\right] \chi_{l} d \rho \\
b_{k k^{\prime}}=\int d \rho \chi_{k} \partial_{\rho} \chi_{k}^{\prime}, C_{k, k^{\prime}, k^{\prime \prime}, k^{\prime \prime \prime}}=\int d \rho \chi_{k} \chi_{k^{\prime}} \chi_{k^{\prime \prime}} \chi_{k^{\prime \prime \prime}} \frac{f(r)}{r^{2}} .
\end{gathered}
$$


The equation 4.55 suggests that there is a solution in a perturbation expansion of powers of $v^{\frac{2}{5}}$. Writing

$$
\tilde{a}_{k}(\tilde{u})=\delta(k) \tilde{\xi}_{0}(\tilde{u})+v^{\frac{2}{5}} \tilde{\eta}_{k}(\tilde{u})+\cdots
$$

we get an equation for $\tilde{\xi}_{0}$,

$$
\tilde{u} \mathcal{J}_{0}-b_{00} \frac{d}{d \tilde{u}} \tilde{\xi}_{0}(\tilde{u})-C_{0000} \tilde{\xi}_{0}(\tilde{u})^{3}=0
$$

and a set of equations for $\tilde{\eta}_{k}$

$$
\begin{aligned}
\tilde{\eta}_{k}(\tilde{u}) & =-\frac{1}{k^{2}}\left(\tilde{u} \mathcal{J}_{k}-b_{k 0} \frac{d}{d \tilde{u}} \tilde{\xi}_{0}(\tilde{u})-C_{k 000} \tilde{\xi}_{0}(\tilde{u})^{3}\right) \\
& \left.=-\frac{1}{k^{2}}\left(\tilde{u}\left(\mathcal{J}_{k}-\mathcal{J}_{0}\right)-\left(b_{k 0}-b_{00}\right) \frac{d}{d \tilde{u}} \tilde{\xi}_{0}(\tilde{u})-\left(C_{k 000}-C_{0000}\right) \tilde{\xi}_{0}(\tilde{u})^{3}\right)\right)
\end{aligned}
$$

In the last line of the above equation we have subtracted the equation for $\chi_{0}(u)$ to make the $k \rightarrow 0$ limit explicit.

If the values of $k$ were discrete, the $k=0$ mode would clearly dominate the dynamics for small $v$. However, $k$ is a continuous parameter starting from zero - this naively indicates that the perturbation expansion in $v^{\frac{2}{5}}$ could break down. Whether this happens or not depends on the behavior of the numerator on the right hand side of 4.58).

It turns out, however, that all the terms like $\left(\mathcal{J}_{k}-\mathcal{J}_{0}\right) \sim k^{2}$ as $k \rightarrow 0$, rendering the perturbation expandion in $v^{\frac{2}{5}}$ well defined. This is shown in Appendix (C).

Thus, in the critical region the solution $\chi_{s}$ is dominated by the zero mode piece proportional to $\tilde{\chi}_{0}$. In terms of the original variables, we have finally

$$
\chi_{s}(\rho, u) \approx v^{\frac{1}{5}} \tilde{\xi}\left(v^{\frac{2}{5}} u\right) \chi_{0}(\rho)+v^{\frac{3}{5}} \int \tilde{\eta}_{k}\left(v^{\frac{2}{5}} u\right) \chi_{k}(\rho) d k
$$

Since the equation for $\tilde{\xi}_{0}$ is identical to the equation for the quantity $\phi$ in the toy model (2.1), we are led to scaling relations identical to the $0+1$ dimensional model. The one point function of the dual operator is given by

$$
<\mathcal{O}>(u) \sim \operatorname{Lim}_{\rho \rightarrow 0}\left[\rho^{1-\Delta_{+}} \chi_{s}(\rho, u)\right]
$$

Therefore, the time dependence of $\mathcal{O}$ is governed by the same scaling behavior.

The above analysis can be carried out for $m^{2}$ away from the critical value as well. In this case, the terms like $\mathcal{J}_{k} \sim k$ as $k \rightarrow 0$, so that the expansion in $v^{5 / 2}$ is not valid. This means that away from the critical point, all the modes are important. 


\subsection{Mass quench}

Another way to quench across the critical point is to consider a time dependent $m^{2}$ with no source, keeping the background geometry fixed. The mass function is taken to asymptote to some constant value of $m^{2}<m_{c}^{2}$ at early times and then rise to a constant value $m^{2}>m_{c}^{2}$ at late times, e.g.

$$
m^{2}(u)=m_{c}^{2}+\left(m_{c}^{2}-m_{1}^{2}\right) \tanh (v u)
$$

Note that we have a mass which depends on the EF coordinate $u$ rather than the time coordinate $t$. The motivation for this is as follows. As mentioned earlier, we may consider the bulk mass term as arising from a coupling of the scalar field with some other field. This latter field acquires an expectation value in response to a source which leads to a mass of our field $\phi$. In this scenario, a time dependent source of the second field would lead, by causality, to an effective mass-squared which has the form $m^{2}(u) G(\rho)+\cdots$, where the ellipsis denote terms which involve $u$ derivatives of $m(u)$. The function $G(\rho)$ vanishes at the boundary and is regular at the horizon. To leading order we therefore get an equation like 4.35 with the potential $V_{0}$ modified by replacing $m^{2} \rightarrow m^{2}(u) G(\rho)$.

The analysis in the previous sections can be now easily repeated for this problem. Below we outline the main steps and results.

Let us consider the case of a time dependent mass which asymptotes to a sufficiently negative value, $m_{1}^{2}$. If the mass remained constant at $m_{1}^{2}$ there is a nonzero static solution $\chi_{0}\left(r, m_{1}\right)$. Now once we make the the mass time dependent we solve (4.35) adiabatically in $\partial_{u}$. For a mass which is slowly varying one expects that the time dependent solution of (3.26) may be constructed around an adiabatic solution,

$$
\chi(\rho, u ; m(u))=\chi_{0}(\rho, m(u))+\epsilon^{2} \chi_{1}(\rho, u)+\cdot .
$$

$\chi_{0}(\rho ; m)$ is the static solution obtained by ignoring the time dependence of the mass completely. Here $\epsilon$ is an adiabaticity parameter which keeps track of the adiabatic expansion.

Following similar arguments like $J$ quench case, the divergence in $\chi_{1}$ can be estimated to yield

$$
\chi_{1}(\rho, u) \sim \frac{1}{\delta m^{2}} \partial_{u}\left(\sqrt{\delta m^{2}(u)}\right)
$$

where $\delta m^{2} \equiv\left(m_{c}^{2}-m^{2}\right)$. The condition for break down of adiabaticity for linear quench $\left(\delta m^{2}(u) \sim v u\right.$ near the critical point) is similar to $0+1$ dim case,

$$
t v^{\frac{1}{2}} \sim 1
$$


When adiabiticity breaks down, the system enters a scaling regime just like the $0+1$ dim case. Following arguments similar to what discussed in the previous section we find,

$$
\chi(\rho, u) \approx v^{\frac{1}{4}} \tilde{\xi}_{0}\left(v^{\frac{1}{2}} u\right) \chi_{0}(\rho)+v^{\frac{3}{4}} \int \tilde{b}_{k}\left(v^{\frac{1}{2}} u\right) \chi_{k}(\rho) d k
$$

The first term defines the scaling relation. The expectation value of the scalar field in the scaling regime has the following properties,

$$
<\mathcal{O}(t)>=<\mathcal{O}\left(t v^{\frac{1}{2}}\right)>v^{\frac{1}{4}}
$$

\section{$5 \quad$ Numerics}

In this section we will solve the equations (3.30) numerically, although our analysis is quite preliminary. Mainly we would like to understand how the system behaves away from the regime of slowly varying quench. It is generally hard to solve PDE's numerically. However we will use the convenient method of lines. In this method the PDE is at first discretized only in the $r$ direction. This gives rise to a set of ODEs. This set of ODEs is then treated in a ODE solver. We fix the boundary condition at the two ends, at the boundary and at the horizon. We use a regularity boundary condition at the horizon and appropriate standard quantization condition at infinity. The initial radial profile of the scalar field and its derivative are given at $t=0$. In most cases the initial value scalar profile would be a solution with $m^{2}=m^{2}\left(t_{0}\right)$. Hence $m^{2}<m_{c}^{2}$. Then we will change $m^{2}$ to some other value higher than the critical mass. The first point to note is that at late times, i.e. out of the critical region, any finite energy excitation is gradually sucked up by the black hole. In fact at late times, the equation can be approximately linearized and the solution in a black hole background can be expressed as a sum of quasi normal modes. Hence, the quasi normal frequency with smallest imaginary part determines the late time decay of the scalar field in a black hole geometry [36]. We find a similar decaying behavior even in the full non-linear theory (Fig 3). Note that the scalar field falls in the black hole as expected.

A time dependent $m(t)$ changes the scalar field profile near the boundary. As the potential vanishes near the black hole horizon, changing the value of $m(t)$ does not have an immediate effect. Hence a time dependent $m(t)$ creates disturbances near the boundary. The disturbances then propagate towards horizon along a light cone. 


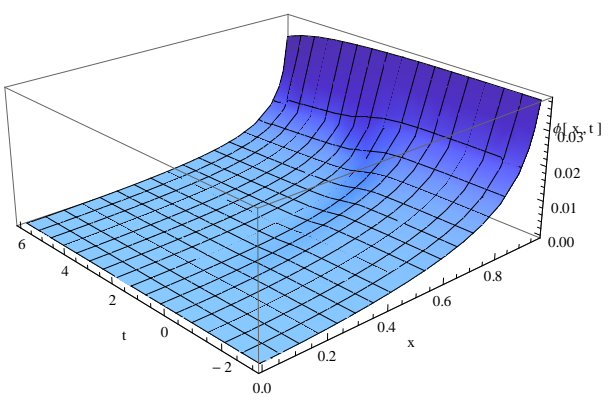

(a) The plot is for $\phi(x, t),\left(x \sim \frac{1}{r}\right)$. It is to be noted how the disturbance propagates following a light cone.

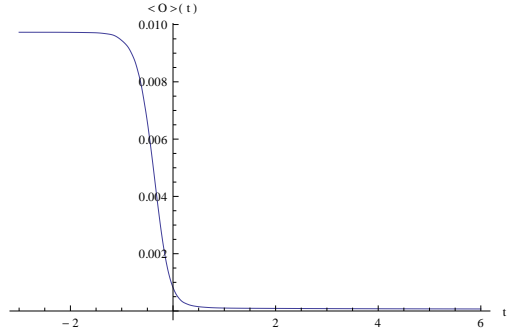

(b) Plot of boundary expectation value $\langle\mathcal{O}\rangle(t)$.

Figure 3: A typical response under a continuous but fast change of $m^{2}(t) \approx-2.1825(0.9-$ $0.1 \tanh (3 t))$. Here $\eta \approx 0.991$ and $m_{c}^{2} \approx-2.169$ at $t=0$.

\section{Outlook}

In this work we have taken a first step in using AdS/CFT methods to study quantum quench across holographic critical points. The particular critical point we study is a mean field transition at any finite temperature, and it is not surprising that the critical behavior can be obtained by a Landau-Ginsburg type model of an appropriate mode. It may appear surprising that the dynamics involves a first order time derivative, even though the underlying theory is Lorentz invariant. As discussed above, this is a feature of non-zero temperature which manifests itself as a dissipative horizon in the bulk. However, similar techniques can be used to study other interesting non-mean field theory transitions. In particular, the zero temperature limit of the transition which we study is of BerezinskiKosterlitz-Thouless type. Other examples of such non-trivial transitions are discussed in [32]. The situation is more subtle in this case : it would be worthwhile to study quench in these systems. It would be also interesting to study dynamics of holographic transitions associated with black branes whose IR geometry is of Lifshitz type 38.

As discussed above, AdS/CFT maps quantum quench in a strongly coupled theory to a classical quench in one higher dimension. The dynamics is completely deterministic, as opposed to e.g. Langevin dynamics - the effect of a non-zero temperature being accounted by the presence of a black brane in the bulk. Such systems have also been studied in the past 31. In these studies, however the interesting questions are rather different. They involve, for example, long time behavior of fluctuations and correlation functions when averaged over initial conditions. These questions can be addressed in our framework as 
well - they would correspond to $1 / N$ corrections of the dual field theory and are left to future work. A related direction is the study of entanglement entropy during a quench which has been studied holographically in other contexts in [9]- [10].

Finally, it would be interesting to extend our considerations to inhomogeneous quench. In this case one interesting question relates to defect formation as one crosses the phase transition. Some aspects of this question has been studied numerically in [37]. It would be interesting to see if expected scaling properties continue to hold, and if there is any analytical insight in terms of the eigenmodes of the radial operator.

\section{A $\quad \alpha=2$ quench}

In this section we will study quantum quench in a simple relativistic Landau-Ginsburg model. The relevant lagranian is 7

$$
\mathcal{L}=-\frac{1}{2} \dot{\phi}^{2}+\frac{1}{2} m^{2}(t) \phi+\frac{1}{4} \phi^{4}+J(t) \phi
$$

In our language this is a system with $\alpha=2$. Quench in a holographic model without any black hole horizon, e.g. a quench in global $A d S$, is expected to obey eqn (22-1) as an effective description. This is suggested by the fact that in this latter situation the lowest order adiabatic correction is proportional to the second derivative of the source, as is consistent with lack of dissipation (see e.g. the last reference in [11]. This kind of model also appears in dynamics of phase transitions in cosmology [39] A thorough discussion on those issues is left to the future.

The lagrangian (A.67) leads to the equation of motion

$$
\frac{d^{2} \phi}{d t^{2}}+m^{2}(t) \phi+\phi^{3}+J(t)=0
$$

When $m^{2}$ and $J$ are time independent, this model has a critical point at $m^{2}=J=0$. We study quench by seting $m^{2}=0$ and considering a time dependent $J(t)=\tanh (v t)$. For small $v$, breakdown of adiabaticity occurs when,

$$
t v^{\frac{1}{4}} \sim 1
$$

The scaling solution in the critical regime is given by,

$$
\phi(t)=\tilde{v}^{\frac{1}{4}} \tilde{\phi}\left(t v^{\frac{1}{4}}\right) .
$$

\footnotetext{
${ }^{7}$ We have rescaled fields to set the self coupling to 1 .
} 
As discussed in the main text this scaling relation fixes $\delta \phi(0)$ and zero crossing time $t_{*}$.

If we continue the quench we may ask how much excess energy (or the equivalently the amplitude of oscillation) remains in the system at late times. If the adiabatic approximation were valid, the excess energy would be suppressed exponentially. However, here the system goes through a critical point where adiabaticity is broken and at late times the energy turns out to scale as a power of $v$.

\section{B $\quad k$ dependence of coefficients : A toy model :}

The purpose of the next two appendices is to justify the perturbation expansion in $v^{2 / 5}$ used to derive the critical region scaling in section 4.2. The idea is to examine the behavior of the eigenfunctions for small values of $k$. Before tackling the problem at hand we discuss a toy model in this appendix. The next appendix discusses the eigenfunctions of the real problem.

The toy model involves the eigenvalue equation

$$
-\partial_{\rho}^{2} \psi_{k}+V(\rho) \psi_{k}=k^{2} \psi_{k}
$$

where the potential is a step function

$$
\begin{aligned}
V(\rho) & =\infty, \rho<0 \\
& =-V_{0} \quad, 0<\rho<1 \\
& =0 \quad, \rho \geq 1
\end{aligned}
$$

We want to find the eigenfunctions $\psi_{k}$ with continuous spectrum, which clearly starts from zero. This potential has features which are similar to the potential $V_{0}(\rho)$ in the text.

The solution for $\rho<1$ is given by

$$
\psi_{k}=\frac{A(k)}{\sqrt{\pi}} \sin \left(\sqrt{k^{2}+V_{0}} \rho\right) \quad 0<\rho<1
$$

The solution for $\rho>1$ is

$$
\psi_{k}=\frac{1}{\sqrt{\pi}} \sin (k \rho+\theta(k))
$$

The $k$-independent coefficient in $(\mathrm{B} .76)$ follows from normalization of the wave function. This may be verified by solving the problem with a IR cutoff at some large value of $\rho=L$, imposing Dirichlet boundary conditions there, and finally taking the limit $L \rightarrow \infty$. 
Even though there is a continuous spectrum starting at $k=0$ the Green's function for the operator on the left hand side of (B.71) exists for generic values of $V_{0}$. However for

$$
\sqrt{V_{0}}=\left(n+\frac{1}{2}\right) \pi \quad n=0,1,2, \cdots
$$

there is a zero mode solution which vanishes at $\rho=0$ and is regular at $\rho=\infty$,

$$
\begin{aligned}
\psi_{0}(\rho) & =\frac{1}{\sqrt{\pi}} \sin \left[\left(n+\frac{1}{2}\right) \pi \rho\right], \quad \rho<1 \\
& =\frac{1}{\sqrt{\pi}}, \quad \rho>1
\end{aligned}
$$

For these values of the potential, the Green's function diverges. The potential for $n=0$ above corresponds to a critical point.

Matching two solutions at $\rho=1$ we get

$$
\begin{array}{r}
A(k) \sin \left(\sqrt{k^{2}+V_{0}}\right)=\sin (k+\theta(k)) \\
A(k) \sqrt{k^{2}+V_{0}} \cos \left(\sqrt{k^{2}+V_{0}}\right)=k \cos (k+\theta(k))
\end{array}
$$

leading to

$$
\begin{aligned}
& A(k)=\frac{k}{\sqrt{k^{2} \sin ^{2}\left(\sqrt{k^{2}+V_{0}}\right)+\sqrt{k^{2}+V_{0}} \cos ^{2}\left(\sqrt{k^{2}+V_{0}}\right)}} \\
& \theta(k)=\tan ^{-1}\left(\frac{k \tan \left(\sqrt{k^{2}+V}\right)}{\sqrt{k^{2}+V}}\right)-k .
\end{aligned}
$$

We now examine the behavior of the wavefunctions in the small $k$ limit. For a generic potential $V_{0} \neq \frac{\pi^{2}}{4}$, the leading order behavior for small $k$ is given by

$$
\begin{aligned}
& A(k) \approx k \frac{1}{\sqrt{V_{0}} \cos \left(\sqrt{V_{0}}\right)}+O\left(k^{2}\right) \\
& \theta(k) \approx k\left(\frac{\tan \left(\sqrt{V_{0}}\right)}{\sqrt{V_{0}}}-1\right)+O\left(k^{3}\right)
\end{aligned}
$$

Now consider a function $J(\rho)$, with finite support. The convolution of $J(\rho)$ with the eigenfunction $\psi_{k}$, in the $k \rightarrow 0$ limit becomes

$$
\begin{aligned}
J_{k} & =A(k) \int_{0}^{1} d k \sin \left(\sqrt{k^{2}+V_{0}} \rho\right) J(\rho)+\int_{1}^{\infty} d k \sin (k \rho+\theta(k)) J(\rho) \\
& \approx A(k) \int_{0}^{1} d k \sin \left(\sqrt{k^{2}+V_{0}} \rho\right) J(\rho)+\int_{1}^{\infty} d k(k \rho+\theta(k)) J(\rho) \\
& \sim O(k)
\end{aligned}
$$


Now let us discuss the critical case $V_{0}=\frac{\pi^{2}}{4}$. In this case we get, upto an overall $k$-independent constant,

$$
\begin{aligned}
& A(k) \approx 1-\frac{k^{2}}{8}+O\left(k^{4}\right) \\
& \theta(k) \approx-\frac{\pi}{2}-\frac{k}{2}+O\left(k^{3}\right)
\end{aligned}
$$

This leads to

$$
\begin{aligned}
J_{k} & =A(k) \int_{0}^{1} d k \sin \left(\sqrt{k^{2}+V_{0}} \rho\right) J(\rho)+\int_{1}^{\infty} d k \sin (k \rho+\theta(k)) J(\rho) \\
& \approx\left(1-\frac{k^{2}}{8}\right) \int_{0}^{1} d k \sin \left(\sqrt{k^{2}+V_{0}} \rho\right) J(\rho)+\int_{1}^{\infty} d k\left(1-\frac{1}{2}(k \rho+k / 2)^{2}\right) J(\rho) \\
& \sim J_{0}+O\left(k^{2}\right)
\end{aligned}
$$

\section{C $k$ dependence of the coefficients : the real problem}

In this appendix we will prove the scaling of quantities like $\left(\mathcal{J}_{k}-\mathcal{J}_{0}\right)$ which appear in (4.58).

The eigenvalue problem we wish to solve is,

$$
-\partial_{\rho}^{2} \psi_{k}(\rho)+V(\rho) \psi_{k}(\rho)=k^{2} \psi_{k}(\rho)
$$

$k$ is a continuous parameter which may be chosen to be positive. We will assume some simple properties for the potential $V(\rho)$ near $\rho=\infty$, i.e., $V(\rho) \sim e^{-\rho}$ as $\rho \rightarrow \infty$. Near the boundary $\rho=0$ the potential $V(\rho)$ is assumed to be sufficiently well behaved so that the above EOM has a solution of the form,

$$
\psi_{k}(0) \sim A \rho^{\alpha_{+}}+B \rho^{\alpha_{-}} .
$$

Where $A$ and $B$ are coefficients of leading and subleading modes. The potential $V(\rho)$ in the problem of interest (i.e. the equation in a black brane background) satisfies these properties. We use the boundary condition,

$$
A=0
$$

The normalization we use is, $\int d \rho \psi_{k}(\rho) \psi_{k^{\prime}}(\rho)=\delta\left(k-k^{\prime}\right)$.

To solve the equation (C.93) for small $k$, we divide the range of $\rho$ in two overlapping parts: (i) in the "far" region $\rho \gg 1$ we may use $V(\rho) \approx-V_{1} \exp (-\rho)$ (ii) in the "near" region we can treat $k^{2}$ purturbatively. Then we match these two solutions in an overlapping region to find a small $k$ solution. 
In the perturbative "far" region:

$$
\psi_{k}(\rho)=A(k)\left(\psi_{0}(\rho)+O\left(k^{2}\right)\right)
$$

Here $\psi_{0}(\rho)$ is the solution of the $k=0$ equation with the correct boundary conditions at $\rho=0$ and $A(k)$ is a constant to be determined. Near $\rho \rightarrow \infty, \psi_{0} \sim a \rho+b$. The boundary condition we choose for the necessary derivative of $\psi_{0}$ is such that $b=1$.

For $\rho \gg 1$ the equation C.93 may be approximated as,

$$
\partial_{\rho}^{2} \psi_{k}(\rho)+\left(V_{1} \exp (-\rho)+k^{2}\right) \psi_{k}(\rho)=0
$$

The real solution of the above equation is given by,

$$
\psi_{k}(\rho)=B_{k} J_{2 i k}\left(2 \sqrt{V_{1} e^{-\rho}}\right)+B_{k}^{*} J_{-2 i k}\left(2 \sqrt{V_{1} e^{-\rho}}\right)
$$

For $\rho \rightarrow \infty$, we have:

$$
\psi_{k} \approx B_{k} \frac{V_{1}^{i k} e^{i k \rho}}{\Gamma(2 i k+1)}+c . c
$$

Demanding that the above solution reaches a delta function normalizable sinusoidal solution we have,

$$
B_{k} B_{k}^{*}=\frac{1}{4} \Gamma(2 i k+1) \Gamma(-2 i k+1)
$$

This condition is needed for the normalization we use and is similar to the choice of coefficient in $(\mathrm{B} .76)$ in the toy problem considered in the previous appendix.

In the small $k$ limit we have a matching region $\left(1 \ll \rho_{0} \ll\left|\log \left(\frac{k^{2}}{V 1}\right)\right|\right)$ where we may expand the Bessel functions in small argument and match with the perturbative solution. We get at lowest order in $k$,

$$
\begin{aligned}
B_{k}+B_{k}^{*} & =A(k) b \\
2 i k\left(B_{k}-B_{k}^{*}\right) & =A(k) a
\end{aligned}
$$

Hence generically for small $k, A(k) \sim k($ note $b=1)$.

Things are special at the critical point, as $a=0$ there. At the critical point $B_{k}=B_{k}^{*}$ and $A(k)=1 \sim o(1)$.

Now consider a function $J(\rho)$ with a compact support such that,

$$
J_{0}=\int J(\rho) \psi_{0}(\rho) d \rho,
$$


is finite. Now generically,

$$
\begin{array}{r}
J_{k}=\int J(\rho) \psi_{k}(\rho) d \rho, \approx A(k) \\
\int J(\rho) \psi_{0}(\rho) d \rho, \\
\sim O(k) \text { as } k \rightarrow 0 .
\end{array}
$$

In contrast, at the critical point,

$$
\begin{aligned}
J_{k} & =\int J(\rho) \psi_{k}(\rho) d \rho, \\
& \approx \int J(\rho)\left(A(k) \psi_{0}(\rho)+o\left(k^{2}\right)\right) d \rho, \\
& \sim J_{0}+o\left(k^{2}\right) \text { as } k \rightarrow 0 .
\end{aligned}
$$

The subleading piece comes from the perturbative $k^{2}$ corrections.

\section{Acknowledgements}

We would like to thank Karl Landsteiner, Satya Majumdar, Gautam Mandal, Shiraz Minwalla, Takeshi Morita, Ganpathy Murthy, Tatsuma Nishioka, Omid Saremi, Alfred Shapere, Tadashi Takayanagi, Sandip Trivedi and especially Kristan Jensen and Krishnendu Sengupta for disucssions. S.R.D. would like to thank Institut de Fisica Teorica at Madrid, Tata Institute of Fundamental Research at Mumbai and Indian Association for the Cultivation of Science at Kolkata for hospitality during the final stages of this work. This work is partially supported by National Science Foundation grants PHY-0970069 and PHY-0855614.

\section{References}

[1] For reviews and references see S. Mondal, D. Sen and K. Sengupta, arXiv:0908.2922; J. Dziarmaga, arXiv:0912.4034; A. Polkovnikov, K. Sengupta,A. Silva and M. Vengalattore, arXiv:1007.5331.

[2] P. Calabrese and J. L. Cardy, J. Stat. Mech. 0504 (2005) P010 arXiv:condmat/0503393|; P. Calabrese and J. L. Cardy, Phys. Rev. Lett. 96 (2006) 136801 arXiv:cond-mat/0601225

[3] P. Calabrese and J. Cardy, arXiv:0704.1880 [cond-mat.stat-mech]]; S. Sotiriadis and J. Cardy, J. Stat. Mech. (2008) P11003, |arXiv:0808.0116 [cond-mat.stat-mech]]; S. Sotiriadis, P. Calabrese and J. Cardy, EPL 87 (2009) 20002, arXiv:0903.0895 [cond-mat.stat-mech]]; S. Sotiriadis and J. Cardy, arXiv:1002.0167 [quant-ph]. 
[4] J. M. Maldacena, Adv. Theor. Math. Phys. 2 (1998) 231 [Int. J. Theor. Phys. 38 (1999) 1113] arXiv:hep-th/9711200];

[5] S. S. Gubser, I. R. Klebanov and A. M. Polyakov, Phys. Lett. B 428, 105 (1998) [arXiv:hep-th/9802109].

[6] E. Witten, Adv. Theor. Math. Phys. 2, 253 (1998) arXiv:hep-th/9802150.

[7] O. Aharony, S. S. Gubser, J. M. Maldacena, H. Ooguri and Y. Oz, Phys. Rept. 323, 183 (2000) [arXiv:hep-th/9905111.

[8] R. A. Janik and R. B. Peschanski, Phys. Rev. D 74, 046007 (2006) arXiv:hepth/0606149|; R. A. Janik, Phys. Rev. Lett. 98, 022302 (2007) arXiv:hepth/0610144|; P. M. Chesler and L. G. Yaffe, Phys. Rev. Lett. 102, 211601 (2009) arXiv:0812.2053 [hep-th]]; P. M. Chesler, L. G. Yaffe, Phys. Rev. D82, 026006 (2010). arXiv:0906.4426 [hep-th]]; S. Bhattacharyya and S. Minwalla, JHEP 0909 (2009) 034 [arXiv:0904.0464 [hep-th]].

[9] J. Abajo-Arrastia, J. Aparicio, E. Lopez, JHEP 1011, 149 (2010). arXiv:1006.4090 [hep-th]];H. Ebrahim, M. Headrick, [arXiv:1010.5443 [hep-th]]; V. Balasubramanian, A. Bernamonti, J. de Boer, N. Copland, B. Craps, E. Keski-Vakkuri, B. Muller, A. Schafer et al., Phys. Rev. Lett. 106, 191601 (2011). arXiv:1012.4753 [hepth]]; V. Balasubramanian, A. Bernamonti, J. de Boer, N. Copland, B. Craps, E. Keski-Vakkuri, B. Muller, A. Schafer et al., Phys. Rev. D84, 026010 (2011). [arXiv:1103.2683 [hep-th]].

[10] T. Albash, C. V. Johnson, New J. Phys. 13, 045017 (2011). arXiv:1008.3027 [hepth]]; T. Takayanagi, T. Ugajin, JHEP 1011, 054 (2010). arXiv:1008.3439 [hep-th]]; C. T. Asplund, S. G. Avery, arXiv:1108.2510 [hep-th]].

[11] S. R. Das, J. Michelson, K. Narayan and S. P. Trivedi, Phys. Rev. D 74, 026002 (2006) arXiv:hep-th/0602107]; A. Awad, S. R. Das, A. Ghosh, J. H. Oh and S. P. Trivedi, Phys. Rev. D 80 (2009) 126011 [arXiv:0906.3275 [hep-th]].

[12] T. Hertog and G. T. Horowitz, JHEP 0407, 073 (2004) arXiv:hep-th/0406134; T. Hertog and G. T. Horowitz, JHEP 0504, 005 (2005) arXiv:hep-th/0503071; N. Turok, B. Craps and T. Hertog, arXiv:0711.1824 [hep-th]; B. Craps, T. Hertog and N. Turok, arXiv:0712.4180 [hep-th].

[13] J. McGreevy and E. Silverstein, JHEP 0508, 090 (2005) arXiv:hep-th/0506130; E. Silverstein, Phys. Rev. D 73, 086004 (2006) arXiv:hep-th/0510044|; G. Horowitz, A. Lawrence and E. Silverstein, JHEP 0907, 057 (2009) [arXiv:0904.3922 [hep-th]].

[14] S. R. Das, T. Nishioka, T. Takayanagi, JHEP 1007, 071 (2010). arXiv:1005.3348 [hep-th]].

[15] K. Hashimoto, N. Iizuka, T. Oka, arXiv:1012.4463 [hep-th]]; K. -Y. Kim, J. P. Shock, J. Tarrio, JHEP 1106, 017 (2011). arXiv:1103.4581 [hep-th]]; S. Prem Kumar, Phys. Rev. D84, 026003 (2011). arXiv:1104.1405 [hep-th]]. S. Janiszewski, A. Karch, [arXiv:1106.4010 [hep-th]]; C. Hoyos, T. Nishioka, A. O'Bannon, arXiv:1106.4030 [hep-th]]. 
[16] U. Gursoy, E. Kiritsis, L. Mazzanti, F. Nitti, Nucl. Phys. B820, 148-177 (2009). arXiv:0903.2859 [hep-th]]. U. Gursoy, E. Kiritsis, L. Mazzanti, F. Nitti, JHEP 1012, 088 (2010). |arXiv:1006.3261 [hep-th]].

[17] J. G. Russo, P. K. Townsend, Class. Quant. Grav. 25, 175017 (2008). arXiv:0805.3488 [hep-th]]; M. Chernicoff and A. Guijosa, JHEP 0806 (2008) 005 arXiv:0803.3070 [hep-th]]; A. Paredes, K. Peeters and M. Zamaklar, JHEP 0904, 015 (2009) |arXiv:0812.0981 [hep-th]]; C. Athanasiou, P. M. Chesler, H. Liu, D. Nickel and K. Rajagopal, arXiv:1001.3880 [hep-th]; E. Caceres, M. Chernicoff, A. Guijosa and J. F. Pedraza, arXiv:1003.5332 [hep-th]; T. Hirata, S. Mukohyama and T. Takayanagi, JHEP 0805 (2008) 089 arXiv:0804.1176 [hep-th]]; T. Hirayama, P. W. Kao, S. Kawamoto and F. L. Lin, arXiv:1001.1289 [hep-th];

[18] A. O'Bannon, JHEP 0901, 074 (2009). arXiv:0811.0198 [hep-th]]; N. Evans, E. Threlfall, arXiv:0807.3679 [hep-th]]; N. Evans, E. Threlfall, Phys. Rev. D79, 066008 (2009). [arXiv:0812.3273 [hep-th]].

[19] N. Iqbal, H. Liu, M. Mezei, Q. Si, Phys. Rev. D82, 045002 (2010). arXiv:1003.0010 [hep-th]].

[20] S. S. Gubser, Phys. Rev. D78, 065034 (2008). [arXiv:0801.2977 [hep-th]].

[21] S. A. Hartnoll, C. P. Herzog, G. T. Horowitz, JHEP 0812, 015 (2008). arXiv:0810.1563 [hep-th]]; S. A. Hartnoll, C. P. Herzog, G. T. Horowitz, Phys. Rev. Lett. 101, 031601 (2008). arXiv:0803.3295 [hep-th]].

[22] P. Basu, A. Mukherjee, H. -H. Shieh, Phys. Rev. D79, 045010 (2009). [arXiv:0809.4494 [hep-th]];

C. P. Herzog, P. K. Kovtun, D. T. Son, Phys. Rev. D79, 066002 (2009). [arXiv:0809.4870 [hep-th]].

[23] S. A. Hartnoll, Class. Quant. Grav. 26 (2009) 224002 [arXiv:0903.3246 [hep-th]]. .

[24] D. Arean, P. Basu, C. Krishnan, JHEP 1010, 006 (2010). arXiv:1006.5165 [hep-th]];

[25] C. P. Herzog, J. Phys. A 42, 343001 (2009) [arXiv:0904.1975 [hep-th]];

[26] For review and further references see T. Faulkner, N. Iqbal, H. Liu, J. McGreevy, D. Vegh, arXiv:1101.0597 [hep-th]];

[27] S. Bhattacharyya, V. EHubeny, S. Minwalla, M. Rangamani, JHEP 0802, 045 (2008). arXiv:0712.2456 [hep-th]];

S. Bhattacharyya, R. Loganayagam, S. Minwalla, S. Nampuri, S. P. Trivedi, S. R. Wadia, JHEP 0902, 018 (2009). [arXiv:0806.0006 [hep-th]].

[28] G. T. Horowitz, V. E. Hubeny, Phys. Rev. D62, 024027 (2000). hep-th/9909056.

[29] G. Policastro, D. T. Son, A. O. Starinets, JHEP 0209, 043 (2002). arXiv:hepth/0205052 [hep-th]];G. Policastro, D. T. Son, A. O. Starinets, JHEP 0212, 054 (2002). [hep-th/0210220]. 
[30] K. Maeda, M. Natsuume, T. Okamura, Phys. Rev. D79, 126004 (2009). arXiv:0904.1914 [hep-th]]

[31] See e.g. A.J. Bray, arXiv:cond-mat/9501089; K. Damle, S. N. Majumdar and S. Sachdev, Phys. Rev. A 54, 5037 (1996) |arXiv:cond-mat/9511058].

[32] K. Jensen, A. Karch, D. T. Son, E. G. Thompson, Phys. Rev. Lett. 105, 041601 (2010); arXiv:1002.3159 [hep-th]];K. Jensen, Phys. Rev. D82, 046005 (2010). |arXiv:1006.3066 |hep-th|; N. Evans, K. Jensen, K. -Y. Kim, Phys. Rev. D82, 105012 (2010). arXiv:1008.1889 [hep-th]].

[33] K. Jensen, private communication.

[34] G.M. Eliashberg, J. E. T. P. Letters 11 (1970) 114.

[35] N. Bao, X. Dong, E. Silverstein, G. Torroba, arXiv:1104.4098 [hep-th]].

[36] R. A. Konoplya, Phys. Rev. D66, 084007 (2002). gr-qc/0207028|. I. Amado, M. Kaminski, K. Landsteiner, JHEP 0905, 021 (2009). [arXiv:0903.2209 [hep-th]].

[37] O. Saremi, private communication

[38] K. Goldstein, S. Kachru, S. Prakash, S. P. Trivedi, JHEP 1008, 078 (2010). arXiv:0911.3586 [hep-th]]. N. Iizuka, N. Kundu, P. Narayan, S. P. Trivedi, arXiv:1105.1162 [hep-th]].

[39] J. Garcia-Bellido, M. Garcia Perez, A. Gonzalez-Arroyo, Phys. Rev. D67, 103501 (2003). [hep-ph/0208228]. 